\title{
Surfaces on Lie Groups, on Lie Algebras, and Their Integrability
}

\author{
A.S. Fokas ${ }^{1,2}$, I.M. Gelfand ${ }^{3}$ \\ (with an Appendix by Juan Carlos Alvarez Paiva) \\ ${ }^{1}$ Department of Mathematical Sciences, Loughborough University of Technology, Loughborough, \\ LE11 3TU, U.K. \\ ${ }^{2}$ Institute for Nonlinear Studies, Clarkson University, Potsdam, New York 13699-5815, USA \\ 3 Department of Mathematics, Rutgers University, New Brunswick, NJ 08903, USA
}

Received: 28 December 1994/in revised form: 7 August 1995

\begin{abstract}
It is shown that the problem of the immersion of a 2-dimensional surface into a 3-dimensional Euclidean space, as well as the $n$-dimensional generalization of this problem, is related to the problem of studying surfaces in Lie groups and surfaces in Lie algebras. A particular case of the general formalism presented here implies that any surface can be characterized in terms of $2 \times 2$ matrices using an arbitrary parametrization. It is also shown that this generality of parametrization is useful for studying integrable surfaces, i.e. surfaces described by integrable equations. In particular starting from a suitable Lax pair (i.e. a suitable integrable equation), it is possible to construct explicitly large classes of integrable surfaces.
\end{abstract}

\section{Introduction}

Let $F=\left(F_{1}, F_{2}, F_{3}\right): \pi \rightarrow \mathbb{R}^{3}$ be an immersion of a domain $\pi \subset \mathbb{R}^{2}$ into the 3-dimensional Euclidean space. Let $(u, v) \in \pi$. The Euclidean metric induces some metric $g_{11}(d u)^{2}+2 g_{12} d u d v+g_{22}(d v)^{2}$ on the surface $F$. If this surface is sufficiently smooth, the functions $g_{i j}(u, v)$, and the functions $d_{i j}(u, v)$ which define the second fundamental form, satisfy a system of three nonlinear equations known as the Gauss-Codazzi equations. These equations are the compatibility condition of the Gauss-Weingarten equations, which are a pair of linear equations characterizing the dependence of the associated Cartan frame on $u$ and $v$. There exist two geometrical characteristics on such a surface known as the Gauss curvature $K$ and the mean curvature $H$.

The question of characterizing surfaces with a given $K$ or a given $H$ has been studied extensively both in the classic and in the recent literature. The most celebrated results in this direction correspond to constant $K$ and to constant $H$. It turns out that the fundamental forms of such surfaces can be expressed in terms of solutions of the sine-Gordon and of the sinh-Gordon equations. These equations are integrable, and hence a large class of their solutions can be given explicitly. Using such global solutions, it is possible to study global properties of the associated 
surfaces (for example the long standing question of Hopf [1], if a compact surface in $\mathbb{R}^{3}$ with constant mean curvature is necessarily a sphere, can be answered [211]). The surfaces with $\partial_{u} \partial_{v}(1 / \sqrt{-K})=0(u, v$ are asymptotic coordinates $)$ [12], with $\partial_{\zeta} \partial_{\bar{\zeta}}(1 / K)=0$ ( $\zeta$ is a conformal coordinate of the second fundamental form) [13], and with $\partial_{\zeta} \partial_{\zeta}(1 / H)=0$ ( $\zeta$ is a conformal coordinate) [13], are also associated with integrable equations.

Following [8] we call a surface integrable, iff its Gauss-Codazzi equations are integrable [14-18]. Integrable equations also arise as the compatibility condition of a pair of linear equations, which in the soliton literature is called a Lax pair [19]. However, in contrast to the Gauss-Weingarten equations, the Lax pair contains a free parameter known as the spectral parameter. (For the integrable surfaces it is possible to insert a spectral parameter in the Gauss-Weingarten equations.) The Gauss-Weingarten equations are usually written in terms of $3 \times 3$ matrices. On the other hand the Lax pairs of the sine-Gordon and sinh-Gordon equations involve $2 \times 2$ matrices. This shows that at least some integrable surfaces can be formulated in terms of $2 \times 2$ matrices. Actually, Bobenko [13], generalizing to arbitrary surfaces some earlier important results of Sym [20] regarding integrable surfaces, has recently shown that if one uses a conformal parametrization then it is possible to write the Gauss-Weingarten equations in terms of $2 \times 2$ matrices.

We will show that the problem of the immersion of a 2-dimensional surface into a 3-dimensional Euclidean space, as well as the $n$-dimensional generalization of this problem, is closely related to the problem of studying surfaces in Lie groups and surfaces in Lie algebras. A particular case of our general theory yields the characterization of an arbitrary surface in terms of $2 \times 2$ matrices (Theorem 2.1). This result, which formulates a surface in terms of an arbitrary parametrization, is a generalization of [13] which uses a conformal parametrization. In spite of the fact that the change of parametrization implies only a gauge transformation and a change of independent variables in the associated Gauss-Weingarten equations, this generality of parametrization is useful for studying geometries described by integrable equations.

Using our formalism it is straightforward to show that in certain cases, it is possible to compute $F$ explicitly: Let $U(u, v, \lambda), V(u, v, \lambda) \in \operatorname{su}(2)$, where $\lambda \in \mathbb{C}$ is the spectral parameter. Assume that $U$ and $V$ satisfy

$$
\frac{\partial U}{\partial v}-\frac{\partial V}{\partial u}+[U, V]=0
$$

Let $\Phi(u, v, \lambda)$ be a solution of the compatible system

Then the function $F \in s u(2)$,

$$
\frac{\partial \Phi}{\partial u}=U \Phi, \quad \frac{\partial \Phi}{\partial v}=V \Phi .
$$

$$
\begin{aligned}
F= & \alpha_{1} \Phi^{-1} U \Phi+\alpha_{2} \Phi^{-1} V \Phi+\alpha_{3} \Phi^{-1} \frac{\partial \Phi}{\partial \lambda}+\alpha_{4} u \Phi^{-1} U \Phi \\
& +\alpha_{5} v \Phi^{-1} V \Phi+\Phi^{-1} M \Phi
\end{aligned}
$$

where $\alpha_{1}, \ldots, \alpha_{5}$ are constant scalars, and $M \in s u(2)$ is a constant matrix, defines an immersion function. The case $\alpha_{1}=\alpha_{2}=\alpha_{4}=\alpha_{5}=M=0$ has been studied by Sym [20].

This implies that starting from a suitable Lax pair (2), it is possible to construct large classes of integrable surfaces. Several such surfaces are studied in [24]. Here 
we only present a simple example: The first and the second fundamental forms of the Weingarten surface

$$
K-U_{2} H+U_{1}^{2}+U_{2}^{2}=0,
$$

where $U_{1}, U_{2}$ are real constants, $U_{1} \neq U_{2}, U_{1} \neq 0$, can be obtained through the solution of the sine-Gordon equation

$$
\frac{\partial^{2} \theta}{\partial u \partial v}+\frac{U_{2}^{2}-U_{1}^{2}}{U_{2}^{2}+U_{1}^{2}} \sin \theta+\frac{2 U_{1} U_{2}}{U_{2}^{2}+U_{1}^{2}} \cos \theta=0 .
$$

This surface is parallel to the surface with $K=-U_{1}^{2}$.

We will also show that the use of Lie point groups [21] provides an effective method for both finding as well as classifying integrable surfaces. As an illustration of this method we will show that the integrable case of inverse harmonic $H$ [13], is distinguished by the fact that this is the only case for which the Gauss-Codazzi equations admit a nontrivial Lie point group of transformations of a given type (see Theorem 3.1).

After this manuscript was written, it was pointed out to the authors by Juan Alvarez that there is a close connection of some of our results with Cartan's method of moving frames [22,23]. Indeed, some of the results of Sects. 1 and 2 are concrete realizations of Cartan's general approach. We note that, in spite of the fact that several examples of Cartan's method are given in [22 and 23], it is in general nontrivial to implement Cartan's construction. A brief description of Cartan's method, as well as the formulation of some of the results of Sects. 1 and 2 in the language of moving frames, is given in the Appendix. This appendix was written by J.C. Alvarez to whom we are grateful.

Global consequences of the results presented here will be discussed elsewhere.

\section{The General Formulation}

Let $G$ be a Lie group and $\mathscr{G}$ the Lie algebra of $G$. We assume that in $\mathscr{G}$ there exists an invariant scalar product, not necessarily positive definite. (Such a scalar product exists for any semi-simple finite dimensional group as well as for many infinite dimensional groups.) We denote the scalar product between $g_{1}$ and $g_{2} \in \mathscr{G}$ by $\left\langle g_{1}, g_{2}\right\rangle$. Since the scalar product is not degenerate there exist an orthonormal basis in $\mathscr{G},\left\langle e_{i}, e_{j}\right\rangle=\delta_{i j}$, where $\delta_{i j}$ denotes the Kroneker delta. In the following presentation we only consider the case that $\operatorname{dim} \mathscr{G}=n<\infty$.

We first introduce a surface in $G$. Let $\Phi(u, v) \in G$ for every $(u, v)$ in some neighborhood of $\mathbb{R}^{2}$. There exists a canonical map (left shift) from the tangent space of $G$ to the Lie algebra $\mathscr{G}$. If $\frac{\partial \Phi}{\partial u}$ and $\frac{\partial \Phi}{\partial v}$ are the tangent vectors of $\Phi$ at the point $(u, v)$, this map is defined by

$$
\frac{\partial \Phi}{\partial u} \Phi^{-1}=U_{j} e_{j}, \quad \frac{\partial \Phi}{\partial v} \Phi^{-1}=V_{j} e_{j}, \quad j=1, \ldots, n
$$

where $U_{j}$ and $V_{j}$ are some functions of $(u, v)$, and throughout this paper summation over repeated indices is assumed. Equations (1.1) define $\Phi$ through its value in the Lie algebra. 
Proposition 1.1. Let $\Phi(u, v) \in G$ be a differentiable function of $u$ and $v$ for every $(u, v)$ in some neighborhood of $\mathbb{R}^{2} . \Phi$ defined by Eqs. (1.1) exists iff the functions $U_{j}$ and $V_{j}$ satisfy

$$
\frac{\partial U_{j}}{\partial v}-\frac{\partial V_{j}}{\partial u}=V_{k} U_{l} c_{k l j}
$$

where $c_{k l j}$ are the structural constants associated with $\mathscr{G}$, i.e.

$$
\left[e_{k}, e_{l}\right]=c_{k l j} e_{j}
$$

In Eqs. (1.2),(1.3) $j=1, \ldots, n$, and summation is assumed over $k$ and $l$, which run from 1 to $n$.

Proof. $\Phi$ exists iff Eqs. (1.1) are compatible, i.e. iff

$$
\frac{\partial}{\partial v}\left(\frac{\partial \Phi}{\partial u} \Phi^{-1}\right)-\frac{\partial}{\partial u}\left(\frac{\partial \Phi}{\partial v} \Phi^{-1}\right)=\left[\frac{\partial \Phi}{\partial v} \Phi^{-1}, \frac{\partial \Phi}{\partial u} \Phi^{-1}\right],
$$

where [,] denotes the usual commutator. This equation together with Eqs. (1.1) and (1.3) imply Eq. (1.2).

We now introduce a surface in $\mathscr{G}$. Let $F(u, v) \in \mathscr{G}$ for every $(u, v)$ in a neighborhood of $\mathbb{R}^{2}$. The first fundamental form of $F$ is defined by

$$
\left\langle\frac{\partial F}{\partial u}, \frac{\partial F}{\partial u}\right\rangle(d u)^{2}+2\left\langle\frac{\partial F}{\partial u}, \frac{\partial F}{\partial v}\right\rangle d u d v+\left\langle\frac{\partial F}{\partial v}, \frac{\partial F}{\partial v}\right\rangle(d v)^{2}
$$

Let $N^{(l)} \in \mathscr{G}, l=1, \ldots, n-2$ be the set of elements defined by

$$
\left\langle N^{(l)}, N^{(l)}\right\rangle=1, \quad\left\langle\frac{\partial F}{\partial u}, N^{(l)}\right\rangle=\left\langle\frac{\partial F}{\partial v}, N^{(l)}\right\rangle=0, \quad l=1, \ldots, n-2 .
$$

The second fundamental forms of $F$ are defined by

$$
\begin{gathered}
\left\langle\frac{\partial^{2} F}{\partial u^{2}}, N^{(l)}\right\rangle(d u)^{2}+2\left\langle\frac{\partial^{2} F}{\partial u \partial v}, N^{(l)}\right\rangle d u d v \\
+\left\langle\frac{\partial^{2} F}{\partial v^{2}}, N^{(l)}\right\rangle(d v)^{2}, \quad l=1, \ldots, n-2
\end{gathered}
$$

We can relate surfaces in $G$ to surfaces in $\mathscr{G}$. Using the adjoint representation we can write

$$
\frac{\partial F}{\partial u}=\Phi^{-1} a_{j} e_{j} \Phi, \quad \frac{\partial F}{\partial v}=\Phi^{-1} b_{j} e_{j} \Phi, \quad j=1, \ldots, n
$$

where $a_{j}$ and $b_{j}$ are some functions of $(u, v)$.

Proposition 1.2. Let $\Phi(u, v) \in G$ be a surface defined by Eqs. (1.1). Let $F(u, v) \in$ $\mathscr{G}$ be a differentiable function of $u$ and $v$ for every $(u, v)$ in some neighborhood 
of $\mathbb{R}^{2}$. Equations (1.7) define a surface $F(u, v) \in \mathscr{G}$ iff the functions $a_{j}$ and $b_{j}$ satisfy

$$
\frac{\partial a_{j}}{\partial v}+a_{k} V_{l} c_{k l j}=\frac{\partial b_{j}}{\partial u}+b_{k} V_{l} c_{k l j}, \quad k, l, j=1, \ldots, n .
$$

Proof. Equations (1.7) are compatible iff

$$
\frac{\partial a_{j}}{\partial v} e_{j}+a_{j}\left[e_{j}, \Phi_{v} \Phi^{-1}\right]=\frac{\partial b_{j}}{\partial u} e_{j}+b_{j}\left[e_{j}, \Phi_{u} \Phi^{-1}\right]
$$

Using (1.1), this equation reduces to (1.8).

We can also use the adjoint representation ${ }^{1}$ to express the elements $N^{(l)} \in \mathscr{G}$ defined in (1.5) in the form

$$
N^{(l)}=\Phi^{-1} c_{j}^{(l)} e_{j} \Phi, \quad l=1, \ldots, n-2, j=1, \ldots, n .
$$

Since the scalar product is invariant under the adjoint representation it is straightforward to compute the first and second fundamental forms of $F$.

Theorem 1.1. Let $a_{j}(u, v), b_{j}(u, v), U_{j}(u, v), V_{j}(u, v), j=1, \ldots, n$, be differentiable functions of $u$ and $v$ for every $(u, v)$ in some neighborhood of $\mathbb{R}^{2}$. Let $\left\{e_{j}\right\}_{j=1}^{n}$ be an orthonormal basis in the Lie algebra $\mathscr{G}$ of the Lie group G. Assume that the functions $a_{j}, b_{j}, U_{j}, V_{j}$ satisfy Eqs. (1.2) and (1.8), where $c_{k l j}, k, l, j=1, \ldots, n$, are the structural constants associated with $\mathscr{G}$. Then

(i) Equations (1.1) define a surface $\Phi(u, v) \in G$.

(ii) Equations (1.7) define a surface $F(u, v) \in \mathscr{G}$, whose first fundamental form is given by

$$
a_{j}^{2}(d u)^{2}+2 a_{j} b_{j} d u d v+b_{j}^{2}(d v)^{2}, \quad j=1, \ldots, n .
$$

Let $c_{j}^{(l)}, 1 \leqq j \leqq n, 1 \leqq l \leqq n-2$ satisfy

$$
\sum_{j=1}^{n} c_{j}^{(l) 2}=1, \quad \sum_{j=1}^{n} a_{j} c_{j}^{(l)}=\sum_{j=1}^{n} b_{j} c_{j}^{(l)}=0, \quad l=1, \ldots, n-2 .
$$

Then the second fundamental forms of $F$ are given by

$$
\begin{gathered}
c_{j}^{(l)}\left(\frac{\partial a_{j}}{\partial u}+a_{k} U_{l} c_{k l j}\right)(d u)^{2}+2 c_{j}^{(l)}\left(\frac{\partial a_{j}}{\partial v}+a_{k} V_{l} c_{k l j}\right) d u d v \\
+c_{j}^{(l)}\left(\frac{\partial b_{j}}{\partial v}+b_{k} V_{l} c_{k l j}\right)(d v)^{2}, \quad l=1, \ldots, n-2 .
\end{gathered}
$$

Proof. The function $\Phi \in G$ defined by (1.1) exists iff Eqs. (1.2) are valid. If $\Phi$ exists, then the function $F \in \mathscr{G}$ defined by (1.7) exists iff Eqs. (1.8) are valid. Using Eqs. (1.7) and (1.9) into (1.4)-(1.6), we find (1.10)-(1.12).

\footnotetext{
1 Throughout this paper we use matrices thus we write $\Phi^{-1} a_{j} e_{j} \Phi$ instead of $A_{d}(\Phi) a_{j} e_{j}$.
} 


\subsection{On the Explicit Computation of the Immersion Function}

It is possible in certain cases, to compute $a_{j}, b_{j}$, and $F$, explicitly.

Theorem 1.2. Let $U_{j}(u, v)$ and $V_{j}(u, v), j=1, \ldots, n$, be differentiable functions of $u$ and $v$ for every $(u, v)$ in some neighborhood of $\mathbb{R}^{2}$. Let $\left\{e_{j}\right\}_{1}^{n}$, be an orthonormal basis in the Lie algebra $\mathscr{G}$ of the Lie group G. Assume that:

(i) $U_{j}$ and $V_{j}$ satisfy $(1.2)$, where $c_{k l j}, k, l, j=1, \ldots, n$, are the structural constants associated with $\mathscr{G}$.

(ii) $U_{j}$ and $V_{j}$ depend on the parameter $\lambda$, but $\lambda$ does not appear explicitly in Eq. (1.2).

Define $U$ and $V$ by

Then,

$$
U=U_{j} e_{j}, \quad V=V_{j} e_{j}
$$

(i) If $A$ and $B$ are defined by

$$
\begin{aligned}
& A=a_{j} e_{j}=\alpha_{1} \frac{\partial U}{\partial u}+\alpha_{2} \frac{\partial U}{\partial v}+\alpha_{3} \frac{\partial U}{\partial \lambda}+\alpha_{4} \frac{\partial}{\partial u}(u U)+\alpha_{5} v \frac{\partial U}{\partial v}+[U, M], \\
& B=b_{j} e_{j}=\alpha_{1} \frac{\partial V}{\partial u}+\alpha_{2} \frac{\partial V}{\partial v}+\alpha_{3} \frac{\partial V}{\partial \lambda}+\alpha_{4} u \frac{\partial V}{\partial u}+\alpha_{5} \frac{\partial}{\partial v}(v V)+[V, M]
\end{aligned}
$$

where $M=m_{j} e_{j}$ and $\alpha_{1}, \ldots, \alpha_{5}, m_{1}, \ldots, m_{n}$ are constant scalars, then the equations

$$
\frac{\partial F}{\partial u}=\Phi^{-1} A \Phi, \quad \frac{\partial F}{\partial v}=\Phi^{-1} B \Phi,
$$

are compatible, and can be used to define a surface $F(u, v) \in \mathscr{G}$.

(ii) The solution of Equations (1.15), where $A$ and $B$ are defined by Eqs. (1.14) is, to within an additive constant matrix, given by

$$
\begin{aligned}
F= & \alpha_{1} \Phi^{-1} U \Phi+\alpha_{2} \Phi^{-1} V \Phi+\alpha_{3} \Phi^{-1} \frac{\partial \Phi}{\partial \lambda}+\alpha_{4} u \Phi^{-1} U \Phi \\
& +\alpha_{5} v \Phi^{-1} V \Phi+\Phi^{-1} M \Phi
\end{aligned}
$$

Proof. (i) The equations

$$
\frac{\partial \Phi}{\partial u}=U \Phi, \quad \frac{\partial \Phi}{\partial v}=V \Phi,
$$

are compatible iff

$$
\frac{\partial U}{\partial v}-\frac{\partial V}{\partial u}+[U, V]=0 .
$$

Equations (1.15) are compatible iff

$$
\frac{\partial A}{\partial v}-\frac{\partial B}{\partial u}+[A, V]+[U, B]=0 .
$$

It can be verified that if $A$ and $B$ are defined by (1.14), and if $U, V$ satisfy Eq. (1.18), then $A$ and $B$ satisfy Eq. (1.19).

(ii) It can be verified that $F$ also satisfies (1.15). Using a variation of parameter it follows that this $F$ is unique to within a constant matrix. 
Remark 1.1. This result is a consequence of the following observation: Equation (1.19) is the variational equation of Eq. (1.18). Indeed, if $U$ and $V$ are replaced by $U+\varepsilon A, V+\varepsilon B$, then the $O(\varepsilon)$ term of Eq. (1.18) yields Eq. (1.19). This means that every symmetry of Eq. (1.18) implies a solution of Eq. (1.19). In particular the invariance of Eq. (1.18) under translations in $u, v, \lambda$ yield the solutions corresponding to $\alpha_{1}, \alpha_{2}, \alpha_{3}$. The solutions corresponding to $\alpha_{4}$ and $\alpha_{5}$ are due to the invariance of Eq. (1.18) under the dilations $u \rightarrow c_{1} u, U \rightarrow c_{1}^{-1} U$ and $v \rightarrow c_{2} v, V \rightarrow c_{2}^{-1} V, c_{1}$ and $c_{2}$ constants. Finally, the solution corresponding to $M$ is due to the invariance of (1.18) under $U \rightarrow D U D^{-1}, V \rightarrow D V D^{-1}, D$ a constant matrix.

\section{The Immersion of a 2-Dimensional Surface in $\mathbb{R}^{3}$}

As an example of the general formalism we consider the case that $G$ is $S U(2)$. Then $e_{j}=-i \sigma_{j}, j=1,2,3$, where

$$
\sigma_{1}=\left(\begin{array}{cc}
0 & 1 \\
1 & 0
\end{array}\right), \quad \sigma_{2}=\left(\begin{array}{cc}
0 & -i \\
i & 0
\end{array}\right), \quad \sigma_{3}=\left(\begin{array}{cc}
1 & 0 \\
0 & -1
\end{array}\right),
$$

and $c_{i j k}=2 e_{i j k}$, where $e_{i j k}, i, j, k=1,2,3$, is the usual antisymmetric tensor.

With the vector $\underline{F}=\left(F_{1}, F_{2}, F_{3}\right)^{T} \in \mathbb{R}^{3}$ we associate the matrix $F=F_{j} e_{j} \in$ $s u(2)$. Thus the problem of the immersion of the 2-dimensional surface $x_{j}=$ $F_{j}(u, v), j=1,2,3$, into the 3-dimensional Euclidean space, becomes the problem of studying the relationship between the 3-dimensional sphere $\Phi(u, v) \in S U(2)$ and the two dimensional surface $F(u, v) \in s u(2)$.

Theorem 2.1. Let $U(u, v)$ and $V(u, v) \in s u(2)$, be differentiable functions of $u$ and $v$ for every $(u, v)$ in some neighborhood of $\mathbb{R}^{2}$. Assume that these functions satisfy the equation

$$
\frac{\partial U}{\partial v}-\frac{\partial V}{\partial u}+[U, V]=0
$$

Then the equations

$$
\frac{\partial \Phi}{\partial u}=U \Phi, \quad \frac{\partial \Phi}{\partial v}=V \Phi,
$$

define a 2-dimensional surface $\Phi(u, v) \in S U(2)$.

Let $A(u, v)$ and $B(u, v) \in s u(2)$ be real differentiable functions of $u$ and $v$ for every $(u, v)$ in some neighborhood of $\mathbb{R}^{2}$. In addition to the above, assume that these functions satisfy

$$
\frac{\partial A}{\partial v}-\frac{\partial B}{\partial u}+[A, V]+[U, B]=0
$$

Then the equations

$$
\frac{\partial F}{\partial u}=\Phi^{-1} A \Phi, \quad \frac{\partial F}{\partial v}=\Phi^{-1} B \Phi,
$$

together with $F=-i F_{j} \sigma_{j}$, i.e.

$$
F=\left(\begin{array}{cc}
-i F_{3} & -F_{2}-i F_{1} \\
F_{2}-i F_{1} & F_{3}
\end{array}\right)
$$


define a 2-dimensional surface $x_{j}=F_{j}(u, v) \in \mathbb{R}, j=1,2,3$, in a 3-dimensional Euclidean space. The first and second fundamental forms of this surface are

$$
\langle A, A\rangle(d u)^{2}+2\langle A, B\rangle d u d v+\langle B, B\rangle(d v)^{2},
$$

and

$$
\left\langle\frac{\partial A}{\partial u}+[A, U], C\right\rangle(d u)^{2}+2\left\langle\frac{\partial A}{\partial v}+[A, V], C\right\rangle d u d v+\left\langle\frac{\partial B}{\partial v}+[B, V], C\right\rangle(d v)^{2},
$$

where

$$
\langle A, B\rangle=-\frac{1}{2} \operatorname{trace}(A B)
$$

and

$$
C=\frac{[A, B]}{|[A, B]|}, \quad|A|=\sqrt{\langle A, A\rangle}
$$

Proof. These results follow from Proposition 1.2, using $e_{j}=-i \sigma_{j}$ and $c_{i j k}=$ $2 e_{i j k}$.

Remark 2.1. Equations (2.2) are the Gauss-Codazzi equations. They are the compatibility conditions of the Gauss-Weingarten equations (2.3). This formulation involves $2 \times 2$ matrices and not $3 \times 3$ matrices.

Remark 2.2. Equations (2.3) can be considered as a Lax pair for the "zero curvature" equations (2.2). However, in order to apply the machinery of the inverse spectral theory one needs to insert a spectral parameter in Eqs. (2.3) (see Sect. 3).

Remark 2.3. Theorem 2.1 characterizes surfaces in terms of a general parametrization. A more restrictive parametrization is used in Theorem 2.2.

Theorem 2.2. Let $U_{1}, U_{2}, V_{1}, a, b_{1}, b_{2}, a \neq 0, b_{2} \neq 0$, be real differentiable functions of $u$ and $v$ for every $(u, v)$ in some neighborhood of $\mathbb{R}^{2}$. Assume that these functions satisfy the Gauss-Codazzi equations (2.2), where $U_{3}, V_{2}, V_{3}$ are defined by

$U_{3}=\frac{\frac{\partial a}{\partial v}-\frac{\partial b_{1}}{\partial u}}{b_{2}}, \quad V_{2}=\frac{b_{1} U_{2}-b_{2} U_{1}}{a}, \quad V_{3}=\frac{b_{1} \frac{\partial a}{\partial v}-b_{1} \frac{\partial b_{1}}{\partial u}-b_{2} \frac{\partial b_{2}}{\partial u}}{a b_{2}}$.

Let $\Phi \in S U(2)$ be defined by Eq. (2.3). Then the equations

$$
\frac{\partial F}{\partial u}=-i \Phi^{-1} a \sigma_{1} \Phi, \quad \frac{\partial F}{\partial v}=-i \Phi^{-1}\left(b_{1} \sigma_{1}+b_{2} \sigma_{2}\right) \Phi,
$$

where $\sigma_{1}, \sigma_{2}, \sigma_{3}$ are the Pauli matrices (2.1), define a 2-dimensional surface $x_{j}=F_{j}(u, v), \quad j=1,2,3$, in $\mathbb{R}^{3}$. Its first and second fundamental forms are

$$
\begin{gathered}
a^{2}(d u)^{2}+2 a b_{1} d u d v+\left(b_{1}^{2}+b_{2}^{2}\right)(d v)^{2}, \\
a U_{2}(d u)^{2}+2 a V_{2} d u d v+\left(b_{1} V_{2}-b_{2} V_{1}\right)(d v)^{2} .
\end{gathered}
$$


This surface is unique to within its position in space. The Gauss and mean curvature are

$$
K=-\left(\frac{U_{1}}{a}\right)^{2}+\frac{U_{2}}{a}\left(\frac{b_{1} U_{1}-a V_{1}}{a b_{2}}\right) \text { and } H=\frac{U_{2}}{a}+\frac{b_{1} U_{1}-a V_{1}}{a b_{2}} .
$$

$A$ frame on this surface is given by $F_{u}, F_{v}$ and $N=-i \Phi^{-1} \sigma_{3} \Phi$.

Proof. These results follow from Theorem 2.1 with the choice $A=-i \alpha \sigma_{1}, B=$ $-i\left(b_{1} \sigma_{1}+b_{2} \sigma_{2}\right), U=-\frac{i}{2} U_{j} \sigma_{j}, V=-\frac{i}{2} V_{j} \sigma_{j}$. In particular Eqs. (2.4) become

$$
a V_{2}+b_{2} U_{1}-b_{1} U_{2}=0, \quad \frac{\partial b_{1}}{\partial u}+b_{2} U_{3}-\frac{\partial a}{\partial v}=0, \quad \frac{\partial b_{2}}{\partial u}+a V_{3}-b_{1} U_{3}=0 .
$$

Solving these equations for $V_{2}, U_{3}, V_{3}$ we find (2.10). The Gauss and mean curvatures are defined by

$$
\begin{gathered}
K=\operatorname{det}\left(I I \cdot I^{-1}\right), \quad H=-\operatorname{trace}\left(I I \cdot I^{-1}\right), \\
I=\left(\begin{array}{ll}
g_{11} & g_{12} \\
g_{21} & g_{22}
\end{array}\right), \quad I I=\left(\begin{array}{ll}
d_{11} & d_{12} \\
d_{21} & d_{22}
\end{array}\right),
\end{gathered}
$$

where $g_{i j}$ and $d_{i j}$ are the entrees of the first and second fundamental forms respectively. Using these definitions together with (2.12) and (2.10b), we find (2.13).

Now we show that the surface $F$ is unique within its position in space. Given the fundamental forms (2.12), Eqs. (2.10) imply $U_{2}, V_{2}, V_{1}$. Since these functions satisfy the Gauss-Codazzi Eqs. (2.2), $\Phi \in S U(2)$ can be defined by Eqs. (2.3) to within three constants. Then Eqs. (2.11) (which are compatible since (2.4) are satisfied), imply $F \in s u(2)$ within three additional constants. These six arbitrary constants correspond to motions of the surface in $\mathbb{R}^{3}$. Indeed, the transformations

$$
\hat{F}=f F f^{-1}+\tilde{A}, \quad \hat{N}=f N f^{-1}, \quad \hat{\Phi}=\Phi f, \quad f \in S U(2), \quad \tilde{A} \in \operatorname{su}(2),
$$

leave Eqs. (2.3) and (2.12) invariant. Furthermore, this transformation introduces six arbitrary constants. The constants of $\tilde{A}$ introduce a translation $\hat{x}_{j}=x_{j}+b_{j}$, while the constants of $f$ introduce a rotation $\hat{x}_{j}=\sum_{k=1}^{3} a_{j k} x_{k}, \sum_{k=1}^{3} a_{l j} a_{l k}=\delta_{l k}$.

All the well known parametrizations can be obtained as particular cases of the above general formulation. The orthogonal and the conformal parametrizations are discussed in Example 2.1 and in Sect. 3.1 respectively.

Example 2.1 (Parametric lines of curvature). Letting $b_{1}=V_{2}=0$, and introducing the notations $b=b_{2}, f=U_{2} / a, h=-V_{1} / b$, the Gauss-Codazzi Eqs. (2.2) become

$$
\begin{gathered}
\frac{\partial}{\partial v}\left(\frac{1}{b} \frac{\partial a}{\partial v}\right)+\frac{\partial}{\partial u}\left(\frac{1}{a} \frac{\partial b}{\partial u}\right)+a b f h=0, \\
\frac{\partial}{\partial u}(b h)-f \frac{\partial b}{\partial u}=0, \quad \frac{\partial}{\partial v}(a f)-h \frac{\partial a}{\partial v}=0 .
\end{gathered}
$$

The first and second fundamental forms are

$$
a^{2}(d u)^{2}+b^{2}(d v)^{2}, \quad a^{2} f(d u)^{2}+b^{2} h(d v)^{2} .
$$


A frame on this surface is

$$
\frac{\partial F}{\partial u}=-i \Phi^{-1} a \sigma_{1} \Phi, \quad \frac{\partial F}{\partial v}=-i \Phi^{-1} b \sigma_{2} \Phi, \quad N=-i \Phi^{-1} \sigma_{3} \Phi
$$

The Gauss and mean curvature of this surface are

$$
K=f h, \quad H=f+h .
$$

Equations (2.15) and (2.16) are the integrability conditions of

$$
\frac{\partial \Phi}{\partial u}=\frac{1}{2}\left(\begin{array}{cc}
-\frac{i}{b} \frac{\partial a}{\partial v} & -a f \\
a f & \frac{i}{b} \frac{\partial a}{\partial v}
\end{array}\right) \Phi, \quad \frac{\partial \Phi}{\partial v}=\frac{1}{2}\left(\begin{array}{cc}
\frac{i}{a} \frac{\partial b}{\partial u} & i b h \\
i b h & -\frac{i}{a} \frac{\partial b}{\partial u}
\end{array}\right) \Phi .
$$

\section{Integrable Surfaces}

We call a surface $F(u, v)$ integrable iff the Gauss-Codazzi equations are integrable. We call an equation integrable iff it can either be linearized directly or it can be solved through the inverse spectral method [14-18]. Equations solvable by this method arise as the compatibility condition of a pair of linear equations known as the Lax pair. This system of linear equations, in contrast to GaussWeingarten equations (2.3), contains a free parameter. This parameter, which will be denoted here by $\lambda$, plays a crucial role in the integration of the associated nonlinear equations.

There exist several methods for inserting a parameter $\lambda$ in Eqs. (2.3). Here we shall emphasize the following method. Suppose that the Gauss-Codazzi equations are invariant under the action of some Lie point group of transformations. If Eqs. (2.3) are not invariant under this group, then the group parameter $\lambda$, will appear in Eqs. (2.3). An elementary illustration of this procedure will be given in Example 3.1 using a well known integrable surface. This method can also be used for a systematic search of integrable surfaces. Here we show, as an example, that the integrable case of harmonic inverse $H$ can be distinguished by the fact that it is the only case that the Gauss-Codazzi equations admit a nontrivial group of transformations of a given type (see Theorem 3.1).

The choice of which local coordinates are used on a surface depends on which particular geometrical property is analyzed. For example, surfaces of given $H$, or given negative $K$, are usually studied using conformal, or asymptotic coordinates, respectively. We illustrate the importance of coordinates in Example 3.2, where we show that in a certain coordinate system, surfaces of $K=0$ are characterized by the Born-Infeld equation.

Theorem 1.2 provides an algorithmic approach of constructing integrable surfaces starting from a suitable Lax pair. Several such surfaces are presented in [24]. An example of a Weingarten surface, and a certain generalization of this Weingarten surface, are given in Example 3.3 and in Example 3.4.

Example 3.1 (Constant $H$ ). The first and second fundamental forms of surfaces of constant mean curvature are

$$
e^{2 G}\left((d u)^{2}+(d v)^{2}\right), \quad \frac{1}{2}\left(H e^{2 G}-1\right)(d u)^{2}+\frac{1}{2}\left(H e^{2 G}+1\right)(d v)^{2},
$$


where $G$ solves the hyperbolic sine-Gordon equation

$$
\frac{\partial^{2} G}{\partial v^{2}}+\frac{\partial^{2} G}{\partial u^{2}}+\frac{1}{4}\left(H^{2} e^{2 G}-e^{-2 G}\right)=0
$$

Indeed, if $H$ is constant, Eqs. (2.16) can be integrated explicitly. It is convenient to write $f$ and $h$ in terms of $H$ and a new variable $G, 2 f=H-e^{-2 G}$, $2 h=H+e^{-2 G}$. Equations (2.15), (2.16) become

$$
\begin{gathered}
a=\alpha(u) e^{G}, \quad b=\beta(v) e^{G} \\
\left(\frac{\alpha(u)}{\beta(v)} G_{v}\right)_{v}+\left(\frac{\beta(v)}{\alpha(u)} G_{u}\right)_{u}+\frac{\alpha(u) \beta(v)}{4}\left(H^{2} e^{2 G}-e^{-2 G}\right)=0
\end{gathered}
$$

where the subscripts $u$ and $v$ denote partial derivative. The functions $\alpha(u)$ and $\beta(v)$ can be taken equal to 1 without loss of generality, since under the change of variables $\frac{1}{\beta(v)} \partial_{v}=\partial_{v^{\prime}}, \frac{1}{\alpha(u)} \partial_{u}=\partial_{u^{\prime}}$, the above differential equation becomes (3.2). Equation (3.2) is the integrability condition of

$$
\begin{aligned}
& \frac{\partial \Phi}{\partial u}=\frac{1}{2}\left(\begin{array}{cc}
-i G_{v} & \frac{1}{2}\left(-H e^{G}+e^{-G}\right) \\
\frac{1}{2}\left(H e^{G}-e^{-G}\right) & i G_{v}
\end{array}\right) \Phi, \\
& \frac{\partial \Phi}{\partial v}=\frac{1}{2}\left(\begin{array}{cc}
i G_{u} & \frac{i}{2}\left(H e^{G}+e^{-G}\right) \\
\frac{i}{2}\left(H e^{G}+e^{-G}\right) & -i G_{u}
\end{array}\right) \Phi .
\end{aligned}
$$

Equation (3.2) is invariant under the orthogonal group

$$
u^{\prime}=u \sin \theta+v \cos \theta, \quad v^{\prime}=u \cos \theta-v \sin \theta
$$

Under the action of this group Eqs. (3.3) become

$$
\begin{aligned}
& \frac{\partial \Phi}{\partial u}=\frac{1}{2}\left(\begin{array}{cc}
i G_{v} & \frac{l}{2}\left(\lambda H e^{G}+\frac{e^{-G}}{\lambda}\right) \\
\frac{i}{2}\left(\frac{H e^{G}}{\lambda}+\lambda e^{-G}\right) & -i G_{v}
\end{array}\right) \Phi, \\
& \frac{\partial \Phi}{\partial v}=\frac{1}{2}\left(\begin{array}{cc}
-i G_{u} & \frac{1}{2}\left(-\lambda H e^{G}+\frac{e^{-G}}{\lambda}\right) \\
\frac{1}{2}\left(\frac{H e^{G}}{\lambda}-\lambda e^{-G}\right) & i G_{u}
\end{array}\right) \Phi,
\end{aligned}
$$

where $\lambda=e^{i \theta}$. Equations (3.4) is the Lax pair of Eq. (3.2).

We note that surfaces of zero mean curvature are characterized by the solution of the Liouville equation

$$
G_{v v}+G_{u u}-\frac{1}{4} e^{-2 G}=0 .
$$

A surface of constant mean curvature corresponding to an elliptic solution of equation (3.2) is given in Fig. 1. 

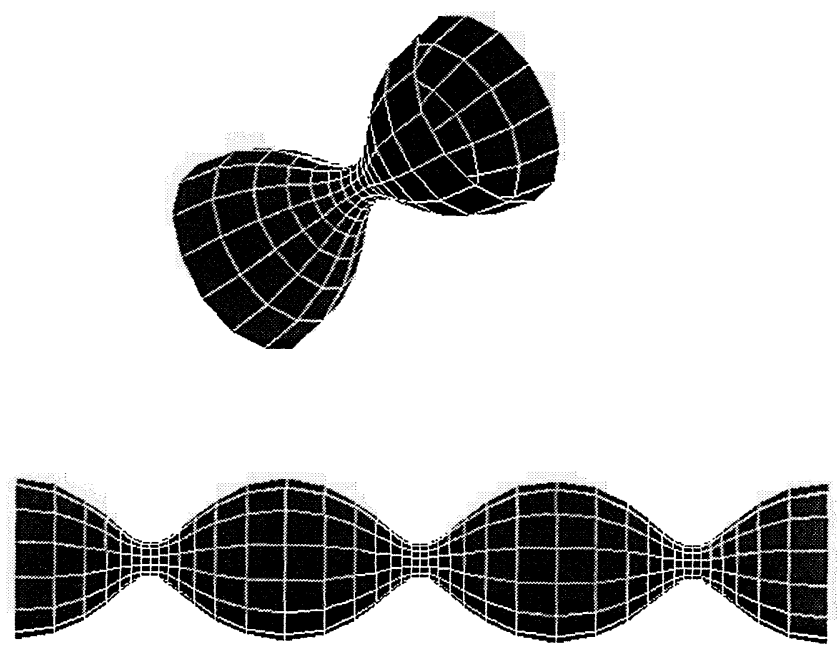

Fig. 1. A surface of constant mean curvature.

Example 3.2 (The Born-Infeld equation). The Born-Infeld equation

$$
\frac{\partial^{2} G}{\partial v^{2}}\left(\frac{\partial G}{\partial u}\right)^{2}+\frac{\partial^{2} G}{\partial u^{2}}\left(\frac{\partial G}{\partial v}\right)^{2}-2 \frac{\partial G}{\partial u} \frac{\partial G}{\partial v} \frac{\partial^{2} G}{\partial u \partial v}=0
$$

characterizes surfaces of zero Gaussian curvature (flat surfaces) in the following coordinates:

$$
(d u)^{2}+(d v)^{2}, \quad e^{G}\left[G_{u}(d u)^{2}+2 G_{v} d u d v+\frac{G_{v}^{2}}{G_{u}}(d v)^{2}\right] .
$$

Indeed, let us consider the frame $F_{u}=-i \Phi^{-1} \sigma_{1} \Phi, F_{v}=-i \Phi^{-1} \sigma_{2} \Phi, N=-i \Phi^{-1}$ $\sigma_{3} \Phi$, i.e. $a=b_{2}=1, b_{1}=0$. Then Eqs. (2.4) become $U_{3}=V_{3}=0, V_{2}=-U_{1}$. The Gauss-Codazzi Eqs. (2.2) yield $V_{1}=-U_{1}^{2} / U_{2}$, and

$$
U_{1_{v}}-V_{1_{u}}=0, \quad U_{2_{v}}-V_{2_{u}}=0 .
$$

These differential equations are solved by $U_{2}=\left(e^{G}\right)_{u}, V_{2}=\left(e^{G}\right)_{v}$, and then the Born-Infled equation follows.

\subsection{The Conformal Parametrization}

The following characterization of surfaces of a given mean curvature $H$ can be found in [13]: Using a conformal parametrization the fundamental forms can be written as

$$
e^{2 G} d \zeta d \bar{\zeta}, \quad D(d \zeta)^{2}+\frac{H}{2} e^{2 G} d \zeta d \bar{\zeta}+\bar{D}(d \bar{\zeta})^{2},
$$

where $H$ is the mean curvature. The bar denotes complex conjugation, $D(\zeta, \bar{\zeta}) \in \mathbb{C}$, $G(\zeta, \bar{\zeta}) \in \mathbb{R}$, and $G, D$ satisfy the equations

$$
\frac{\partial^{2} G}{\partial \zeta \partial \bar{\zeta}}-e^{-2 G}|D|^{2}+\left(\frac{H}{4}\right)^{2} e^{2 G}=0, \quad \frac{\partial D}{\partial \bar{\zeta}}=\frac{1}{4} e^{2 G} \frac{\partial H}{\partial \zeta} .
$$


These equations are the compatibility conditions of

$$
\frac{\partial \Psi}{\partial \zeta}=\left(\begin{array}{cc}
\frac{\partial G}{\partial \zeta} & -D e^{-G} \\
\frac{H}{4} e^{G} & 0
\end{array}\right) \Psi, \quad \frac{\partial \Psi}{\partial \bar{\zeta}}=\left(\begin{array}{cc}
0 & -\frac{H}{4} e^{G} \\
\bar{D} e^{-G} & \frac{\partial G}{\partial \bar{\zeta}}
\end{array}\right) \Psi .
$$

This result also follows from Theorem 2.2 with the identification $\zeta=u+i v$, $b_{1}=0, \quad a=b_{2}=e^{G}, \quad U_{2}=-2 D_{R} e^{-G}+H e^{G} / 2, \quad V_{2}=-2 D_{I} e^{-G}, \quad D=D_{R}+i D_{I}$, $\Psi=\Phi \exp (G / 2)$.

Theorem 3.1. Equations (3.9), which are the Gauss-Codazzi equations with respect to conformal coordinates of a surface of mean curvature $H$, admit a Lie-point group of transformations of the type

$G^{\lambda}=G+\lambda \mathrm{g}(\zeta, \bar{\zeta}, D, \bar{D}, G)+0\left(\lambda^{2}\right), \quad D^{\lambda}=D+\lambda \mathrm{D}(\zeta, \bar{\zeta}, D, \bar{D}, G)+0\left(\lambda^{2}\right)$,

where $\mathfrak{g}, \mathfrak{D}$ are arbitrary differential functions of the arguments indicated, iff

$$
\frac{1}{H}=f(\zeta)+\bar{f}(\zeta)
$$

The associated group of transformations is given by

$$
\begin{gathered}
H^{\lambda}=(1+i \lambda f)(1-i \lambda \bar{f}) H, \quad G^{\lambda}=G-\ln [(1+i \lambda f)(1-i \lambda \bar{f})], \\
D^{\lambda}=\frac{D}{(1+i \lambda f)^{2}} .
\end{gathered}
$$

In this case the Lax pair of Eqs. (3.9) is

$$
\begin{aligned}
& \frac{\partial \Psi}{\partial \zeta}=\left(\begin{array}{cc}
G_{\zeta}-\frac{\lambda f_{\zeta}}{1+\lambda f} & -\left(\frac{1-\lambda \bar{f}}{1+\lambda f}\right) D e^{-G} \\
\frac{H}{4} e^{G} & 0
\end{array}\right) \Psi, \\
& \frac{\partial \Psi}{\partial \bar{\zeta}}=\left(\begin{array}{cc}
0 & -\frac{H}{4} e^{G} \\
\left(\frac{1+\lambda f}{1-\lambda \bar{f}}\right) \bar{D} e^{-G} & G_{\bar{\zeta}}+\frac{\lambda \bar{f}_{\bar{\zeta}}}{1-\lambda \bar{f}}
\end{array}\right) \Psi .
\end{aligned}
$$

Proof. Substituting

$$
G^{\lambda}=G+\lambda \mathrm{g}, \quad D^{\hat{\lambda}}=D+\lambda \mathfrak{D}, \quad H^{\hat{\lambda}}=H+\lambda \mathfrak{h},
$$

where $\mathfrak{h}(\zeta, \bar{\zeta})$ is an arbitrary differentiable function, in Eqs. (3.9) we find

$$
\begin{gathered}
\mathfrak{g}_{\zeta \bar{\zeta}}+\frac{H^{2}}{8} e^{2 G} \mathfrak{g}+\frac{H}{8} e^{2 G} \mathfrak{h}-D \overline{\mathfrak{d}} e^{-2 G}-\mathfrak{D} \bar{D} e^{-2 G}+2|D|^{2} e^{-2 G} \mathfrak{g}=0, \\
\mathfrak{d}_{\bar{\zeta}}=\frac{\mathfrak{h}_{\zeta}}{4} e^{2 G}+\frac{H_{\zeta}}{2} e^{2 G} \mathfrak{g},
\end{gathered}
$$

where $\mathfrak{g}_{\zeta \bar{\zeta}}$ and $\mathfrak{d}_{\bar{\zeta}}$ denote total derivatives. We next use the assumption that $\mathfrak{g}$ and $\mathfrak{d}$ depend only on $\zeta, \bar{\zeta}, D, \bar{D}, G$. For example, the total derivative of $\mathrm{D}$ with respect to $\bar{\zeta}$ equals

$$
\frac{\partial \mathfrak{d}}{\partial \bar{\zeta}}+\frac{\partial \mathfrak{d}}{\partial D} D_{\bar{\zeta}}+\frac{\partial \mathfrak{d}}{\partial \bar{D}} \bar{D}_{\bar{\zeta}}+\frac{\partial \mathfrak{d}}{\partial G} G_{\bar{\zeta}}
$$


Substituting this expression and a similar one for $\grave{D}_{\zeta \bar{\zeta}}$ in Eqs. (3.15) and (3.16), and using Eqs. (3.9) to replace $\frac{\partial^{2} G}{\partial \zeta \partial \tilde{\zeta}}, \frac{\partial D}{\partial \tilde{\zeta}}, \frac{\partial \bar{D}}{\partial \zeta}$, we find two equations involving $\mathfrak{g}, \mathfrak{D}, \mathfrak{h}, G, D, H$, their derivatives, as well as

$$
\bar{D}_{\bar{\zeta}}, G_{\zeta}, G_{\bar{\zeta}}, G_{\zeta} G_{\bar{\zeta}}, G_{\bar{\zeta}} D_{\zeta}
$$

Equating the coefficients of the quantities (3.17) to zero in these equations we obtain certain constraints for $\mathfrak{g}, \mathfrak{d}$ and $\mathfrak{h}$ :

The coefficients of $\bar{D}_{\bar{\zeta}}: \frac{\partial \mathfrak{d}}{\partial \bar{D}}=\frac{\partial \mathfrak{D}}{\partial G}=0$ or $\mathfrak{D}=\mathfrak{D}(\zeta, \bar{\zeta}, D)$.

The coefficients of $G_{\bar{\zeta}}: \frac{\partial \grave{D}}{\partial \bar{\zeta}} e^{-2 G}+\frac{\partial \grave{D}}{\partial G} \frac{H_{\zeta}}{4}=\frac{\mathfrak{h}_{\zeta}}{4}+\frac{H_{\zeta}}{2} \mathfrak{g}$.

The coefficients of $G_{\zeta} G_{\bar{\zeta}}: \frac{\partial^{2} \mathrm{~g}}{\partial G^{2}}=0$.

Thus

$$
\mathfrak{D}=\mathfrak{d}(\zeta, D), \quad \mathfrak{g}=\mathfrak{g}(\zeta, \bar{\zeta}, D) .
$$

The coefficients of $G_{\zeta}, G_{\bar{\zeta}} D_{\zeta}$ together with (3.18) imply

$$
\begin{gathered}
\frac{\partial^{2} \mathfrak{g}}{\partial \bar{\zeta} \partial D}=0, \quad \frac{\partial^{2} \mathfrak{g}}{\partial^{2} D} H_{\zeta}=0, \quad \frac{\partial \mathfrak{g}}{\partial D} \mathfrak{h}_{\zeta}=0, \quad \frac{\partial^{2} \mathfrak{g}}{\partial \zeta \partial \bar{\zeta}}=0, \\
\frac{\partial^{2} \mathfrak{g}}{\partial \zeta \partial D} \frac{H_{\zeta}}{4}+\frac{\partial \mathfrak{g}}{\partial D} \frac{H_{\zeta \zeta}}{4}+\frac{H^{2}}{2} \mathfrak{g}+\frac{H}{8} \mathfrak{h}=0, \\
D \overline{\mathfrak{D}}+\mathfrak{D} \bar{D}-2|D|^{2} \mathfrak{g}=0, \\
\frac{\partial \mathfrak{D}}{\partial D} H_{\zeta}=\mathfrak{h}_{\zeta}+2 H_{\zeta} \mathfrak{g} .
\end{gathered}
$$

Equation (3.21) implies that $\frac{\overline{\mathcal{D}}(\bar{\zeta}, \bar{D})}{\bar{D}}$ is independent of $\bar{D}$, thus

$$
\mathfrak{d}(\zeta, D)=F(\zeta) D, \quad \mathfrak{g}=\frac{1}{2}(F+\bar{F})=\mathfrak{g}(\zeta, \bar{\zeta}) .
$$

Then (3.20) yields

$$
H^{2} \mathfrak{g}+H \mathfrak{h}=0, \quad \text { or } \quad \mathfrak{h}=-H G=-\frac{1}{2}(F+\bar{F}) H .
$$

These equations together with (3.22) imply

$$
F H_{\zeta}=\mathfrak{h}_{\zeta}+2 H_{\zeta} G \quad \text { or } \quad(\bar{F}-F) H_{\zeta}=F_{\zeta} H .
$$

Thus

$$
\{\ln (\bar{F}-F) H\}_{\zeta}=0, \quad \text { or } \quad H=\frac{M(\bar{\zeta})}{\bar{F}-F} .
$$

Since $H \in \mathbb{R}, M(\bar{\zeta})$ is purely imaginary, i.e. $M(\bar{\zeta})=i \lambda, \lambda \in \mathbb{R}$. Thus

$$
H=\frac{2 i \lambda}{\bar{F}-F}, \quad \mathfrak{d}=F(\zeta) D, \quad \mathfrak{g}=\frac{1}{2}(F+\bar{F}), \quad \mathfrak{h}=i \lambda \frac{F+\bar{F}}{F-\bar{F}} .
$$


Letting $F=-2 i \lambda f$, these equations reduce to

$$
H=\frac{1}{f+\bar{f}}, \quad \mathfrak{h}=i \lambda \frac{f-\bar{f}}{f+\bar{f}}, \quad \mathfrak{d}=-2 i \lambda f D, \quad \mathfrak{g}=i \lambda(\bar{f}-f) .
$$

Equations (3.23) are the infinitesimal versions of Eqs. (3.13).

Equations (3.14) can be derived from (3.10) using Eqs. (3.13).

\subsection{An Integrable Weingarten Surface and Generalizations}

A particular case of Theorem 1.2 implies the following integrable Weingarten surface.

Example 3.3. Let $\theta(u, v)$ be a solution of the sine-Gordon equation

$$
\frac{\partial^{2} \theta}{\partial u \partial v}+\frac{U_{2}^{2}-U_{1}^{2}}{U_{2}^{2}+U_{1}^{2}} \sin \theta+\frac{2 U_{1} U_{2}}{U_{2}^{2}+U_{1}^{2}} \cos \theta=0,
$$

$U_{1}, U_{2}$ constants, $U_{1} \neq 0, U_{1} \neq U_{2}$. Then the surface with fundamental forms

$$
\begin{gathered}
(d u)^{2}+\frac{2 \cos \theta}{U_{1}^{2}+U_{2}^{2}} d u d v+\frac{(d v)^{2}}{\left(U_{1}^{2}+U_{2}^{2}\right)^{2}}, \\
U_{2}(d u)^{2}+\frac{2\left(U_{2} \cos \theta-U_{1} \sin \theta\right)}{U_{1}^{2}+U_{2}^{2}} d u d v+\frac{U_{2}(d v)^{2}}{\left(U_{1}^{2}+U_{2}^{2}\right)^{2}},
\end{gathered}
$$

is the Weingarten surface,

$$
K-U_{2} H+\left(U_{1}^{2}+U_{2}^{2}\right)=0 .
$$

The Gaussian and mean curvature of this surface are

$$
K=U_{2}^{2}-U_{1}^{2}+2 U_{1} U_{2} \cot \theta, \quad H=2 U_{2}+2 U_{1} \cot \theta .
$$

Proof. Rather than using Theorem 1.2 we verify this result directly: Letting $a=$ $1, U_{1}$ constant, $U_{2}$ constant, Eqs. (2.10) yield

$$
V_{2}=b_{1} U_{2}-b_{2} U_{1}, \quad U_{3}=-\frac{b_{1 u}}{b_{2}}, \quad V_{3}=-\frac{b_{2} b_{2 u}+b_{1} b_{1 u}}{b_{2}} .
$$

The solution of two of the Gauss-Codazzi equations (2.2) yields

$$
b_{1}=\frac{\cos \theta}{U_{1}^{2}+U_{2}^{2}}, \quad b_{2}=\frac{\sin \theta}{U_{1}^{2}+U_{2}^{2}}, \quad V_{1}=-\frac{\sin \theta U_{2}+\cos \theta U_{1}}{U_{1}^{2}+U_{2}^{2}} .
$$

Then the third Gauss-Codazzi equation (2.2) becomes (3.24).

We note that if $U_{2}=0$, then the above theorem characterizes surfaces of constant negative Gauss curvature $\left(K=-U_{1}^{2}\right)$.

The following generalization of this result is proven in [24].

Example 3.4. Let $w(u, v)$ be a solution of the sine-Gordon equation

$$
\frac{\partial^{2} w}{\partial v^{2}}-\frac{\partial^{2} w}{\partial u^{2}}+\frac{1}{2} \sin 2 w=0
$$


Let the subscripts $u$ and $v$ denote partial derivatives. Let $R(u, v)$ be a solution of the linear equation

$$
\left(a_{3} R_{v}+a_{4} R_{u}+a_{5} R\right)_{u}+a_{1}\left(a_{3} R_{v}+a_{4} R_{u}+a_{5} R\right)+a_{2} R=0,
$$

where

$$
\begin{gathered}
a_{1}=-w_{u} \tan w+\left(\varphi_{u}-w_{v}\right) \tan \varphi, \quad a_{2}=\left(\varphi_{v}-w_{u}\right) \tan w+\left(w_{v}-\varphi_{u}\right) \tan \varphi \\
a_{0} a_{3}=-\sin w, \quad a_{0} a_{4}=\cos w \tan \varphi, \\
a_{0} a_{5}=\left[\varphi_{u}+(\tan \varphi)^{2} w_{v}-w_{v}\right] \cos w+\left(\varphi_{v}-2 w_{u}\right) \sin w \tan \varphi, \\
a_{0}=\left(2 \varphi_{u}+w_{v}\right) \frac{(\cos \varphi)^{2}}{\cos w},
\end{gathered}
$$

and $\varphi(u, v)$ is an arbitrary function such that $a_{0} \neq 0$. Then

$$
\begin{gathered}
R^{2}(\sin w)^{2}(d u)^{2}+2 \rho R^{2} \sin \theta \sin w d u d v+\rho^{2} R^{2}(d v)^{2}, \\
-\left[R(\sin w)^{2} \cos \varphi(d u)^{2}+R \sin \varphi \sin 2 w d u d v+\rho R \sin \theta \sin (\varphi+w)(d v)^{2}\right],
\end{gathered}
$$

where $\theta$ and $\rho$ are given by

$$
\rho=\frac{\sin \varphi \cos w}{\sin (\varphi+\theta)}, \quad \tan \theta=-\tan \varphi+\frac{\tan \varphi}{a_{3} \frac{R_{v}}{R}+a_{4} \frac{R_{u}}{R}+a_{5}},
$$

are the first and second fundamental forms of the surface

$$
K+\frac{\cos \varphi}{R} H+\frac{1}{R^{2}}=0 .
$$

Acknowledgements. We thank J.C. Alvarez for several important remarks, A. Bobenko for illuminating discussions, Q.M. Liu for computations used in Theorem 3.1, and M. Heil for the plots. This work was partially supported by the National Science Foundation under grant numbers DMS9111611, DMS-9204075, and by the Air Force Office of Scientific Research under grant number F49620-93-1-0088. I.M. Gelfand's visit to U.K. was made possible by the generous support of the Royal Society, and of the London Mathematical Society.

\section{Appendix}

\section{by Juan Carlos Alvarez Paiva}

The purpose of this appendix is to relate some of the results of the main paper to Cartan's method of moving frames. We start with a short and, necessarily, incomplete, review of the method; for details and examples see [22] and [23].

Let $H$ be a homogeneous space for a Lie group $G$. If $\Sigma$ is a 2-dimensional manifold consider the right action of $G$ on the space of smooth maps from $\Sigma$ to $H$, $C^{\infty}(\Sigma, H)$. The main problem addressed by Cartan is the classification of the orbits of $G$ on $C^{\infty}(\Sigma, H)$.

Cartan's simple and beautiful idea is summarized in the following proposition

Proposition 1. If on a G-invariant subset, $C$, of $C^{\infty}(\Sigma, H)$ we can define a map,

such that:

$$
\varphi: C \rightarrow C^{\infty}(\Sigma, G),
$$

a) $\pi \circ \varphi(f)=f$, if $f$ is an element of $C$ and $\pi$ is the canonical projection of $G$ onto $H$. 
b) $\varphi(f \cdot g)=\varphi(f) \cdot g$, for all $g \in G$.

Then the Lie algebra valued 1-form $d \varphi(f) \cdot \varphi(f)^{-1}$ depends only on the orbit of $G$ through $f$.

Proof. The proof is just the following simple computation:

$$
\begin{aligned}
d \varphi(f g) \cdot \varphi(f g)^{-1} & =d(\varphi(f) \cdot g)(\varphi(f) \cdot g)^{-1} \\
& =d \varphi(f) \cdot g \cdot g^{-1} \varphi(f)^{-1}=d \varphi(f) \cdot \varphi(f)^{-1} .
\end{aligned}
$$

Note that the 1-form $d \varphi(f) \cdot \varphi(f)^{-1}$ is the pullback of the Maurer-Cartan form on $G$ under the map $\varphi(f)$. As such, it satisfies the equation

$$
d \omega=\frac{1}{2}[\omega, \omega] .
$$

Any Lie algebra-values 1-form on $\Sigma$ satisfying this equation is the pullback of the Maurer-Cartan form under some map from $\Sigma$ to $G$, but this map is not necessarily of the form $\varphi(f)$ for some $f$ in $C$.

In what follows we shall call the map $\varphi(f)$ a Cartan lift of $f$.

We now indicate how the general results of Sects. 1 and 2 can be formulated in the language of moving frames. We follow the notation of Sect. 1.

Let $G$ be a Lie group and $\mathscr{G}$ its Lie algebra. Let $G$ act on $\mathscr{G}$ via the adjoint representation; form the semidirect product, $G \bowtie \mathscr{G}$. If the pair of $\mathscr{G}$-valued one forms,

$$
\begin{aligned}
& \left(\sum U_{j} e_{j}\right) d u+\left(\sum V_{j} e_{j}\right) d v, \\
& \left(\sum a_{j} e_{j}\right) d u+\left(\sum b_{j} e_{j}\right) d v,
\end{aligned}
$$

is considered as a $\mathscr{G} \bowtie \mathscr{G}$-valued form, then Eqs. (1.2) and (1.8) imply that this form satisfies the Maurer-Cartan equations for $G \bowtie \mathscr{G}$. We have then a map, $\mathscr{F}$, from a neighbourhood of the origin in $R^{2}$ to $G \bowtie \mathscr{G}$ such that the pullback of the Maurer-Cartan form under $\mathscr{F}$ equals the $\mathscr{G} \bowtie \mathscr{G}$-valued form. This map is uniquely defined up to right translation. In the notation of Sect. 1, the $G$-component of $\mathscr{F}$ is $\Phi$, and the $\mathscr{G}$-component is $F$.

If one starts with a map,

$$
F: \Sigma \rightarrow \mathscr{G},
$$

then, considering $\mathscr{G}$ as a homogeneous space for the group $G \bowtie \mathscr{G}$, one can construct a Cartan lift,

$$
\varphi(F): \Sigma \rightarrow G \bowtie \mathscr{G} .
$$

The $G$-component of $\phi_{F}$ is a surface on the Lie group associated to $F$.

In Sect. $2, \mathbb{R}^{3}$ is identified with $s u(2)$ and the action of the Euclidean group is identified with the action of $S U(2) \bowtie s u(2)$. Given a surface, $F: \Sigma \rightarrow s u(2)$, a Cartan lift,

$$
\varphi(F)=(\Phi, F): \Sigma \rightarrow S U(2) \bowtie s u(2),
$$

is defined by Eqs. (2.11).

The pullback of the Maurer-Cartan form of $S U(2)$ under $\Phi$ gives the second fundamental form of the surface. Furthermore the Maurer-Cartan equation it satisfies are the Gauss-Codazzi compatibility conditions. 


\section{References}

1. Hopf, H.: Differential Geometry in the Large. Lect. Notes Math., Vol. 1000, Berlin, Heidelberg, New York: Springer, 1983

2. Wente, H.C.: Counterexample to a Conjecture of H. Hopf. Pac. J. Math. 121, 193-243 (1986)

3. Abresch, U.: Constant Mean Curvature Tori in Terms of Elliptic Functions. J. Reine Angew. Math. 394, 169-192 (1987)

4. Spruck, J.: The Elliptic Sinh-Gordon Equation and the Construction of Toroidal Soap Bubbles. In: Hildebrandt, S., Kinderlehrer, D., Miranda, M. (eds.) Calculus of Variations and Partial Differential Equations. Proceedings, Tronto 1986, Lect. Notes Math., Vol. 1340, Berlin, Heidelberg, New York: Springer, 1988, pp. 273-301

5. Walter, R.: Explicit Examples to the H-Problem of Heinz Hopf. Geom. Dedicate 23, 187-213 (1987)

6. Wente, H.C.: Twisted Tori of Constant Mean Curvature in $\mathbb{R}^{3}$. In: Tromba, A. (ed.) Seminar on New Results in Nonlinear Partial Differential Equations, Aspects Math. Vol. E10, Braunschweig Wiesbaden: Vieweg, 1987, pp. 1-36

7. Pinkall, U., Sterling, I.: On the Classification of Constant Mean Curvature Tori. Ann. Math. 130, 407-451 (1989)

8. Bobenko, A.I.: Integrable Surfaces. Funkts. Anal. Prilozh. 24 (3), 68-69 (1990)

9. Abresch, U.: Old and New Periodic Solutions of the Sinh-Gordon Equation. In: Tromba, A. (ed.) Seminar on New Results in Nonlinear Partial Differential Equations, Aspects Math. Vol. E10, Braunschweig Wiesbaden: Vieweg, 1987, pp. 37-73

10. Hitchin, N.S.: Harmonic Maps from a 2-Torus to the 3-Sphere. J. Diff. Geom. 31, 627-710 (1990)

11. Bobenko, A.I.: Math. Ann. 290, 209-245 (1991)

12. Levi, D., Sym, A.: Phys. Lett. 149A, 381-387 (1990)

13. Bobenko, A.I.: Surfaces in Terms of 2 by 2 Matrices, Old and New Integrable Cases. In: Harmonic Maps and Integrable Systems, Fordy, A.P., Wood, J.C. (eds.), Aspects of Mathematics, Vieweg, 1994

14. Novikov, S.P.: (ed.) Theory of Solitons. New York: Consultants Bureau, 1984

15. Ablowitz, M.J., Segur, H.: Solitons and the Inverse Scattering Transformation. SIAM Stud. Appl. Mat. 4, Philadelphia: SIAM, 1981

16. Newell, A.C.: Solitons in Mathematics and Physics, CBMS-NSF Regional Conf. Ser. in Appl. Math. 48, Philadelphia: SIAM, 1985

17. Faddeev, L.D., Takhtajan, V.E.: Hamiltonian Methods in the Theory of Solitons. Berlin, Heidelberg, New York: Springer, 1986

18. Fokas, A.S., Zakharov, V.E.: (eds.), Important Developments in Soliton Theory. Berlin, Heidelberg, New York: Springer, 1993

19. Lax, P.: Comm. Pure. Appl. Math. 21, 467 (1968)

20. Sym, A.: Soliton Surfaces and Their Applications (Soliton Geometry from Spectral Problems). In: Geometrical Aspects of the Einstein Equations and Integrable Systems, Lect. Notes Phys., Vol. 239, Berlin, Heidelberg, New York: Springer, 1985, pp. 154-231

21. Olver, P.J.: Applications of Lie Groups to Differential Equations. Berlin, Heidelberg, New York: Springer-Verlag, 1986

22. Cartan, E.: La Theorie des Groups Finis et Continus et la Geometrie Differentielle Traitees par la Methode du Repere Mobile. Editions Jaeques Gabay, 1992

23. Griffiths, P.: On Cartan's method of Lie groups and moving frames as applied to uniqueness and existence questions in differential geometry. Duke Math. J. (1974)

24. Fokas, A.S., Gel'fand, I.M., Liu, Q.M.: The motion of curves, of surfaces, and their integrability. Preprint, 1995

Communicated by A. Jaffe 